\title{
Red cell distribution width (RDW): a prognostic indicator of severe COVID-19
}

\author{
Changzheng Wang ${ }^{1 \#}$, Hongmei Zhang ${ }^{2 \#}$, Xiaocui $\mathrm{Cao}^{2 *}$, Rongrong Deng ${ }^{3}$, Yi Ye ${ }^{4}$, Zhongxiao Fu ${ }^{1}$, \\ Liyao Gou ${ }^{4}$, Feng Shao ${ }^{5}, J^{\prime} \mathrm{Li}^{4}$, Weiyang $\mathrm{Fu}^{6}$, Xiaomei Zhang ${ }^{4}$, Xiao Ding ${ }^{1}$, Jianping Xiao ${ }^{4}$, \\ Chuanjian $\mathrm{Wu}^{4}$, Tao $\mathrm{Li}^{1}$, Huan $\mathrm{Qi}^{4}$, Chengbin $\mathrm{Li}^{1}$, Zhongxin $\mathrm{Lu}^{2}$
}

${ }^{1}$ Department of Laboratory Medicine, The Second Clinical Medical College, Yangtze University, Jingzhou, China; ${ }^{2}$ Department of Laboratory Medicine, the Central Hospital of Wuhan, Tongji Medical College, Huazhong University of Science and Technology, Wuhan, China; ${ }^{3}$ Pediatric Medical Center, The Second Clinical Medical College, Yangtze University, Jingzhou, China; ${ }^{4}$ Clinical Department (IVD), Shenzhen Mindray Bio-Medical Electronics Co., Ltd, Shenzhen, China; ${ }^{5}$ Department of Intensive Care Unit (ICU), The Second Clinical Medical College, Yangtze University, Jingzhou, China; ${ }^{6}$ Department of Respiratory Medicine, Jingzhou Infectious Disease Hospital, Jingzhou, China

Contributions: (I) Conception and design: Y Ye, L Go, J Li, X Zhang, F Shao; (II) Administrative support: H Zhang, R Deng; (III) Provision of study materials or patients: C Wu, T Li; (IV) Collection and assembly of data: Z Fu, W Fu, X Ding; (V) Data analysis and interpretation: C Wang, H Zhang, X Cao; (VI) Manuscript writing: All authors; (VII) Final approval of manuscript: All authors.

\#These authors contributed equally to this work.

Correspondence to: Professor Huan Qi. Clinical Department (IVD), Shenzhen Mindray Bio-Medical Electronics Co., Ltd, Shenzhen, China. Email: qihuan@mindray.com; Professor Chengbin Li. Department of Laboratory Medicine, Jingzhou Central Hospital, The Second Clinical Medical College, Yangtze University, Jingzhou, China. Email: jzlcb002@163.com; Professor Zhongxin Lu. Department of Laboratory Medicine, the Central Hospital of Wuhan, Tongji Medical College, Huazhong University of Science and Technology, Wuhan, China. Email: lzx71@yahoo.com.

Background: The global mortality rate for coronavirus disease 2019 (COVID-19) is 3.68\%, but the mortality rate for critically ill patients is as high as $50 \%$. Therefore, the exploration of prognostic predictors for patients with COVID-19 is vital for prompt clinical intervention. Our study aims to explore the predictive value of hematological parameters in the prognosis of patients with severe COVID-19.

Methods: Ninety-eight patients who were diagnosed with COVID-19 at Jingzhou Central Hospital and Central Hospital of Wuhan, Hubei Province, were included in this study.

Results: The median age of the patients was 59 [28-80] years; the median age of patients with a good prognosis was 56 [28-79] years, and the median age of patients with a poor outcome was 67 [35-80] years. The patients in the poor outcome group were older than the patients in the good outcome group $(\mathrm{P}<0.05)$. The comparison of hematological parameters showed that lymphocyte count (Lym\#), red blood cells (RBCs), hemoglobin (HGB), hematocrit (HCT), mean corpuscular volume (MCV), and mean corpuscular hemoglobin $(\mathrm{MCH})$ were significantly lower in the poor outcome group than in the good outcome group $(\mathrm{P}<0.05)$. Further, the red cell volume distribution width-CV $(\mathrm{RDW}-\mathrm{CV})$ and red cell volume distribution width-SD (RDW-SD) were significantly higher in the poor outcome group than in the good outcome group $(\mathrm{P}<0.0001)$. Receiver operating characteristic (ROC) curves showed RDW-SD, with an area under the ROC curve (AUC) of 0.870 [95\% confidence interval (CI) 0.796-0.943], was the most significant single parameter for predicting the prognosis of severe patients. When the cut-off value was 42.15 , the sensitivity and specificity of RDW-SD for predicting the prognosis of severe patients were $73.1 \%$ and $80.2 \%$, respectively. Reticulocyte (RET) channel results showed the RET level was significantly higher in critical patients than in moderate patients and severe patients $(\mathrm{P}<0.05)$, which may be one cause of the elevated RDW in patients with a poor outcome.

Conclusions: In this study, the hematological parameters of COVID-19 patients were statistically analyzed. RDW was found to be a prognostic predictor for patients with severe COVID-19, and the increase in RET may contribute to elevated RDW. 
Keywords: Red cell distribution width (RDW); novel coronavirus (SARS-Cov-2); COVID-19; receiver operating characteristic (ROC); linear discriminant analysis (LDA); prognostic indicator

Submitted Jul 27, 2020. Accepted for publication Sep 28, 2020.

doi: $10.21037 /$ atm-20-6090

View this article at: http://dx.doi.org/10.21037/atm-20-6090

\section{Introduction}

Severe acute respiratory syndrome coronavirus 2 (SARS$\mathrm{CoV}-2)$ is the third deadly coronavirus type and the cause of coronavirus disease 2019 (COVID-19) (1). With the spread of the COVID-19 epidemic, the number of infected people has escalated. As of April 27, 2020, 185 countries and regions were involved in the COVID-19 pandemic, with over 2,970,000 diagnosed cases and over 200,000 deaths (mortality rate, approximately 7.0\%) reported worldwide (2). The main manifestations of patients with COVID-19 are fever, dry cough, and fatigue. In severe cases, dyspnea or hypoxemia usually occurs 1 week after disease onset, and patients may rapidly become critically ill. According to an observational study on patients with COVID-19 in Wuhan, among 52 critically ill patients, 32 patients $(61.5 \%)$ died within 28 days of onset, and the median time from intensive care unit (ICU) admission to death was 7 days [interquartile range (IQR) 3-11], showing a high mortality rate for critical patients (3). Therefore, it is essential to monitor the dynamic condition of patients diagnosed with COVID-19 during hospitalization, especially to predict the prognosis of patients in advance so that clinical intervention can be implemented as early as possible.

As hematological tests can supply the most common and easy-to-get diagnosis and treatment evidence, they are widely used in medical institutions at all levels. Because of low detection costs and high automation, hematological tests have become the first choice for disease monitoring and the evaluation of general conditions. Some hematological parameters transform into patients with COVID-19. Upon disease aggravation in patients with COVID-19, the lymphocyte count (Lym\#) decreases significantly; the Lym\# in patients who die is significantly lower than that in survivors (4-7). Also, some studies have shown the red cell volume distribution width (RDW) of patients with severe COVID-19 increases significantly, showing that the role of erythroid cell parameters as risk indicators may be underestimated $(8,9)$. It has also been reported the platelet (PLT) count of patients with severe COVID-19 increases significantly (10). Although many studies on COVID-19 have reported hematological parameters, most have only focused on the differences in hematological parameters between patients with different severities of COVID-19. Only a few studies have reported the predictive role of hematological parameters in the prognosis of patients with severe COVID-19. Therefore, we retrospectively analyzed the hematology parameters of inpatients with severe COVID-19 at our hospital to explore the value of hematological parameters in predicting the prognosis of severe patients, and found that RDW is a prognostic predictor for patients with severe COVID-19. We present the following article in accordance with the MDAR reporting checklist (available at http://dx.doi. org/10.21037/atm-20-6090).

\section{Methods}

\section{Patients}

This study included 98 patients with COVID-19 at Jingzhou Central Hospital and Central Hospital of Wuhan from January 20, 2020, to March 30, 2020. The trial was divided into two phases: prognosis analysis and mechanism analysis. For the prognosis analysis, the epidemiological information, clinical information, and hematological test results retrieved using a hematology analyzer (no test results from the reticulocyte (RET) channel) for 43 patients who were diagnosed as severe at admission from January 20, 2020, to March 10, 2020, were collected. According to whether the patients became critically ill during hospitalization, they were divided into a good outcome group $(\mathrm{N}=31)$ and a poor outcome group $(\mathrm{N}=12)$. In the mechanism analysis, 55 patients with COVID-19 from March 12, 2020, to March 30, 2020, were included to study the mechanism in positive results for the prognosis analysis; their hematological test results retrieved using a hematology analyzer (including test results from the RET channel) were collected to classify patients according to the Guidelines for the Diagnosis and Treatment of COVID-19 (Seventh Edition) 
issued by the National Health Commission of the People's Republic of China. The numbers of moderate, severe, and critical cases were 40, 5, and 10, respectively. For all patients, viral detection confirmed COVID-19 using the SARS-CoV-2 nucleic acid detection kit (fluorescence polymerase chain reaction (PCR), Shanghai BioGerm Medical Biotechnology Co., Ltd.).

COVID-19 severity was classified according to the Guidelines for the Diagnosis and Treatment of COVID-19 (Seventh Edition) issued by the National Health Commission of the People's Republic of China:

(I) Mild: cases were classified as mild cases when patients had mild clinical symptoms and no imaging manifestations of pneumonia;

(II) Moderate: cases were classified as moderate when patients had a fever, respiratory tract symptoms, and imaging manifestations of pneumonia;

(III) Severe: cases were classified as severe when adult patients met any of the following criteria: (i) respiratory rate $(\mathrm{RR}) \geq 30$ times/min, (ii) resting oxygen saturation (finger) $\leq 93 \%$, (iii) partial pressure of oxygen $\left(\mathrm{PaO}_{2}\right)$ /fraction of inspired oxygen $\left(\mathrm{FiO}_{2}\right)$ $\leq 300 \mathrm{mmHg}$, and (iv) $>50 \%$ lesion progression within $24-48 \mathrm{~h}$ detected by lung imaging;

(IV) Critical: cases were classified as critical when patients met one of the following criteria: a. respiratory failure and the need for mechanical ventilation, $b$. shock, c. custodial care in the ICU because of organ failure other than lung failure.

\section{Laboratory data collection}

One hundred seventy-three hematological test results were collected from the 98 patients. For the prognosis analysis, 102 hematological test results were collected, including complete blood count parameters [white blood cell (WBC), red blood cell (RBC), platelet (PLT), etc.], WBC classification parameters [neutrophil count (Neu\#), lymphocyte count (Lym\#), monocyte count (Mon\#), etc.] and cell morphological parameters [red cell volume distribution width-CV (RDW-CV), red cell volume distribution width-SD (RDW-SD), mean corpuscular volume (MCV), etc.]. According to whether the patients became critical within 3 days after sample collection, the test results were divided into the poor outcome group (26 cases) and the good outcome group (76 cases). If a patient was already critically ill before sample collection, the test results were not included in the statistics.
Seventy-one hematological test results were collected for the mechanism analysis. The data from the above hematological test results and RET channel data were collected: RET percent (\%), reticulocyte count (RET\#), high fluorescence reticulocyte ratio (HFR\%), and middle fluorescence reticulocyte ratio (MFR\%), etc. The blood samples were divided into the moderate group (46 cases), severe group (6 cases), and critical group (19 cases) according to each patient's condition at the time of sample collection.

Hematological tests were performed using a Mindray BC-6800 automatic hematology analyzer (Mindray, Shenzhen, China).

All procedures performed in this study involving human participants were in accordance with the Declaration of Helsinki (as revised in 2013). The Ethics Committee approved this study of Jingzhou Central Hospital and the Ethics Committee of the Central Hospital of Wuhan. As this study was a retrospective study, there was no patient's private data, including patient name, ID number, telephone, and address, were involved. Only demographic information and laboratory testing data of patients were collected and analyzed, so there is no informed consent in this study.

\section{Statistical analysis}

Continuous variables are expressed as the mean or median, and categorical variables are presented as the percentage of composition ratio. Between-group comparisons were performed using Student's $t$-test. The Multigroup comparisons were performed using a one-way analysis of variance, and later pair-wise comparisons were performed using the Tukey-Kramer procedure. Categorical variables were compared using Pearson's chi-square test. Receiver operating characteristic (ROC) curves were used to analyze the classification performance of the parameters. Single parameters were combined into pairs using linear discriminant analysis (LDA), pairs with intergroup differences were screened, and their classification performance was analyzed using ROC curves. $\mathrm{P}<0.05$ was considered statistically significant. GraphPad Prism version 8.0 was used to perform the above statistical analysis.

\section{Results}

\section{Presentation characteristics}

The epidemiological information, clinical information, 
Table 1 Presentation characteristics

\begin{tabular}{|c|c|c|c|c|}
\hline Characteristics & Total $(\mathrm{N}=43)$ & $\begin{array}{l}\text { Good outcome } \\
\qquad(\mathrm{N}=31)\end{array}$ & $\begin{array}{l}\text { Poor outcome } \\
\qquad(\mathrm{N}=12)\end{array}$ & $P$ value \\
\hline Age, median [range] & 59 [28-80] & 56 [28-79] & $67[35-80]$ & 0.036 \\
\hline Gender & & & & 0.987 \\
\hline Male & $25(58.1)$ & $18(58.1)$ & $7(58.3)$ & \\
\hline \multicolumn{5}{|l|}{ Exposure history, n (\%) } \\
\hline Close contact with suspected cases within 2 weeks & $4(9.3)$ & $2(6.5)$ & $2(16.7)$ & 0.308 \\
\hline Close contact with confirmed cases within 2 weeks & $6(14.0)$ & $4(12.9)$ & $2(16.7)$ & $>0.999$ \\
\hline \multicolumn{5}{|l|}{ Comorbidities, n (\%) } \\
\hline Malignancy & $1(2.3)$ & $1(3.2)$ & 0 & $>0.999$ \\
\hline Cerebrovascular disease & $4(9.3)$ & $2(6.5)$ & $2(16.7)$ & 0.308 \\
\hline COPD & 0 & 0 & 0 & \\
\hline Chronic kidney disease & $2(4.7)$ & 0 & $2(16.7)$ & 0.073 \\
\hline Viral hepatitis & 0 & 0 & 0 & \\
\hline \multicolumn{5}{|l|}{ Signs and symptoms, n (\%) } \\
\hline Fever & $36(83.7)$ & $28(80.7)$ & $8(95.0)$ & 0.060 \\
\hline Headache & $1(2.3)$ & $1(3.2)$ & 0 & $>0.999$ \\
\hline
\end{tabular}

$P$ value shows the differences between good outcome groups and poor outcome groups. $\mathrm{P}<0.05$ was considered statistically significant. COPD, chronic obstructive pulmonary disease.

symptoms, and signs of patients in the good outcome group (31 patients) and poor outcome group (12 patients) were compared and analyzed (Table 1). The median age of the patients was 59 years (range 28-80 years). There was a significant difference $(\mathrm{P}=0.036)$ between the median age of the patients in the good outcome group (56 years, range 28-79 years) and the patients in the poor outcome group (67 years, range $35-80$ years). However, the epidemiological history, comorbidities, symptoms, and signs were not significantly different between the two groups of patients $(\mathrm{P}>0.05)$.

\section{Comparison of differences in Hematological test results for severe patients with different outcomes}

From the comparison of the hematological test results of blood samples from the good outcome group and poor outcome group (Table 2), the Lym\#, RBC, hemoglobin (HGB), hematocrit (HCT), MCV, and mean corpuscular hemoglobin $(\mathrm{MCH})$ in the poor outcome group were significantly lower than those in the good outcome group $(\mathrm{P}<0.05)$, and the RDW-CV. Further, RDW-SD in the poor outcome group were significantly higher than those 
Table 2 Comparison of hematology analysis results of severe patients with different prognosis

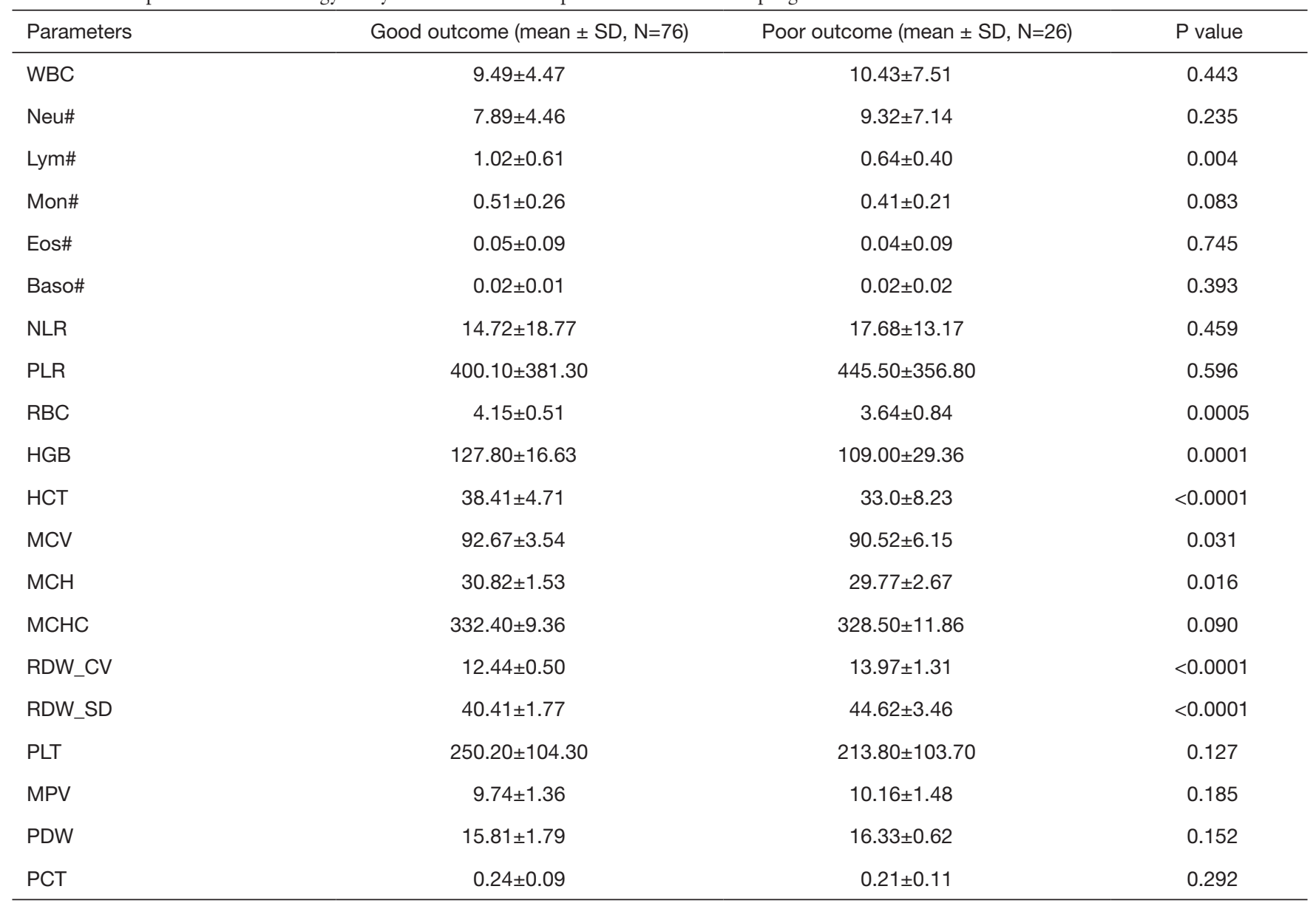

Data are shown as mean \pm SD. $P$ value indicates the difference between the good outcome group and the poor outcome group. $\mathrm{P}<0.05$ was considered statistically significant. WBC, white blood cells; Neu\#, neutrophil count; Lym\#, lymphocyte count; Mon\#, monocyte count; Eos\#, eosinophil count; Baso\#, basophil count; NLR, neutrophil-lymphocyte ratio; PLR, platelet-lymphocyte ratio; RBC, red blood cells; $\mathrm{HGB}$, hemoglobin; HCT, hematocrit; MCV, mean corpuscular volume; $\mathrm{MCH}$, mean corpuscular hemoglobin; MCHC, mean corpuscular hemoglobin concentration; RDW-CV, red cell volume distribution width-coefficient of variation; RDW-SD, red cell volume distribution width-standard deviation; PLT, platelet; MPV, mean platelet volume; PDW, platelet volume distribution width; PCT, plateletcrit.

in the good outcome group $(\mathrm{P}<0.05)$. For the other routine hematological parameters involved in the statistical analysis, including WBC, Neu\#, Mon\#, Eos\#, and PLT, there were no significant differences between the two groups $(\mathrm{P}>0.05)$.

\section{Hematological test results predict the prognosis of severe patients}

ROC curves were used to analyze the routine hematological parameters significantly different between the good outcome group and the poor outcome group. The ROC curves of some single parameters are shown in Figure 1A. The minimum sum of squares of the false positive rate and the false-negative rate was used as the reference for the choice of the optimal cut-off value. The results showed that RDW-SD was the best single parameter for predicting the prognosis of severe patients, with an area under the ROC curve (AUC) of 0.870 [ $95 \%$ confidence interval (CI) $0.796-0.943$ ]. When the cut-off value was 42.15 , the sensitivity and specificity of RDW-SD for predicting the prognosis of severe patients were $73.1 \%$ and $80.2 \%$, respectively (Table 3).

LDA was used to linearly combine any two single parameters from all hematological test results to generate new joint parameters. Among them, Lym\# \& RDW-CV, Lym\# \& RDW-SD, and HGB \& RDW-SD had the highest AUCs for predicting the prognosis of severe COVID-19 

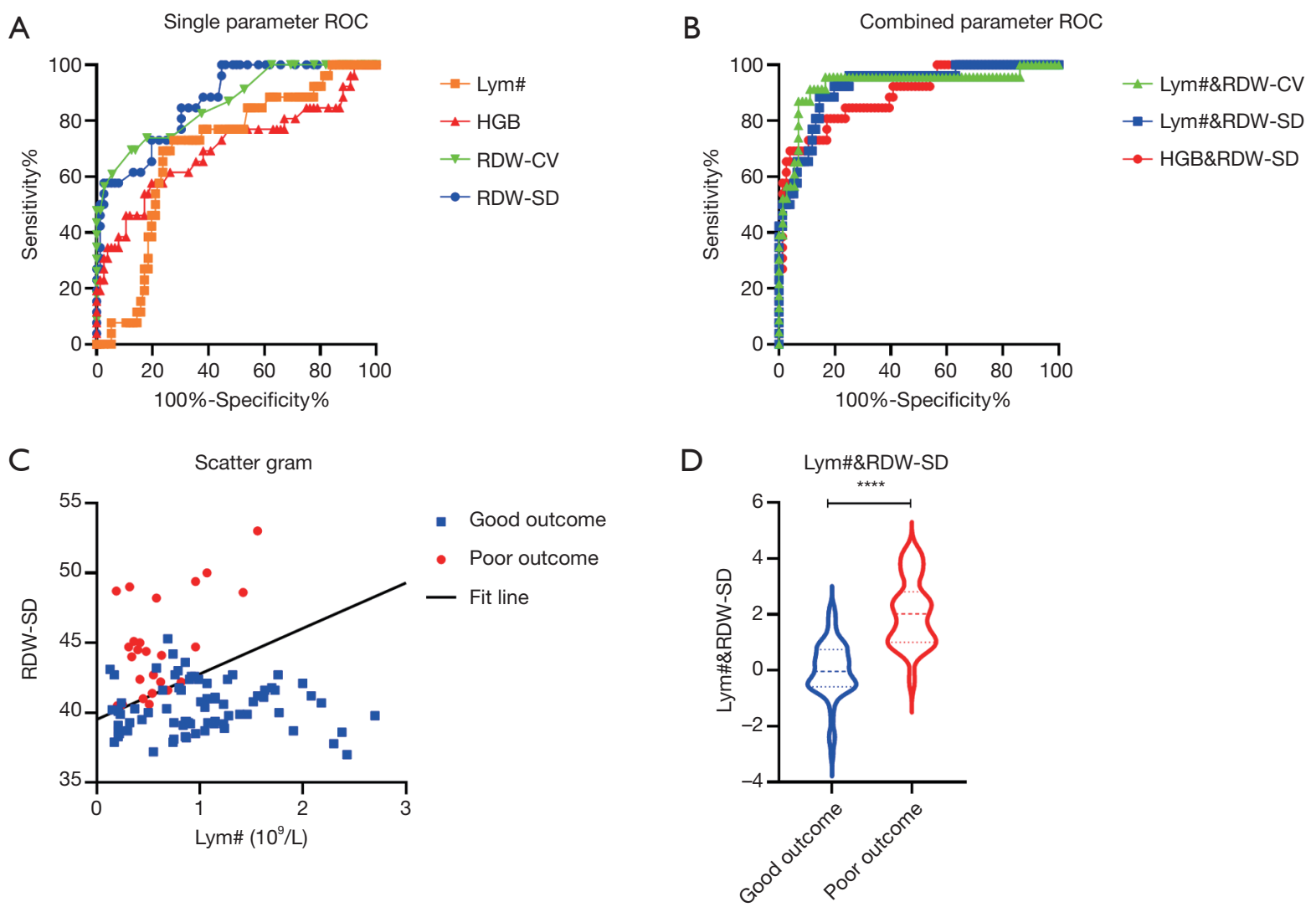

Figure 1 Prediction analysis of hematology parameters and the outcomes of patients with severe COVID-19. (A) ROC curve, the single parameter for predicting the prognosis of ill patients; (B) ROC curve, joint parameters for predicting the prognosis of ill patients; (C) the linearly fitted schematic scatter plot for Lym\# \& RDW-SD; (D) comparison of Lym\# \& RDW-SD in ill patients with different prognoses. Lym\# \& RDW-SD: joint parameter generated after linear fitting of Lym\# and RDW-SD. **** $\mathrm{P}<0.0001$.

patients; the ROC curves are shown in Figure $1 B$. Taking Lym\# \& RDW-SD as an example, a scatter plot for blood samples from severe patients with different outcomes, using Lym\# as the abscissa and RDW-SD as the ordinate, is shown in Figure 1C. The fitted line is the best mode of the joint parameter for the differentiation of the two sets of data. Between-group comparisons showed that Lym\# \& RDW-SD was significantly higher in the poor outcome group than in the good outcome group $(\mathrm{P}<0.0001)$ (Figure 1D). The AUC for Lym\# \& RDW-SD for predicting the prognosis of severe patients was 0.920 (95\% CI $0.860-0.979)$. When the cut-off value was 0.92 , the sensitivity and specificity of Lym\# \& RDW-SD for predicting the prognosis of severe patients were $88.5 \%$ and $85.5 \%$, respectively (Table 3). When the Lym\# \& RDW$\mathrm{SD}$ of severe patients were more significant than or equal to 0.92 , there was a high probability that these patients would develop critical illness within 3 days; when the value was less than 0.92 , the probability of patients developing critical illness within 3 days was low.

\section{RET channel test results for patients with different severities of COVID-19}

To investigate the cause of the significant increase in RDW in severe patients with a poor outcome, blood samples from COVID-19 patients from March 12, 2020, to March 30, 2020, were used for a mechanism analysis. A scatter plot of data obtained using a hematology analyzer showed as disease severity increased, the RET\% increased, which might be an explanation for the increased RDW in severe patients with a poor outcome. The RBC volume/hemoglobin concentration (V/HC) scattergram showed that the magenta scatters of critical patients were significantly left-skewed, indicating that RETs with a low HC increased significantly, which may represent a particular pattern of erythroid 
Table 3 Prediction analysis of hematology parameters of patients with severe COVID-19

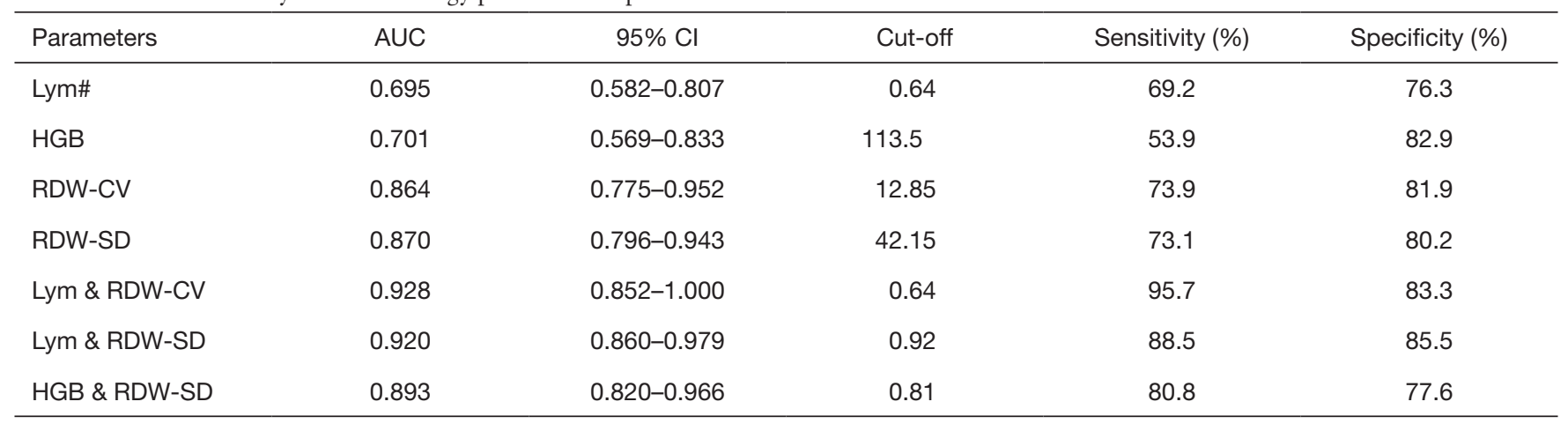

AUC, area under the ROC curve; $95 \% \mathrm{Cl}, 95 \%$ confidence interval.

A
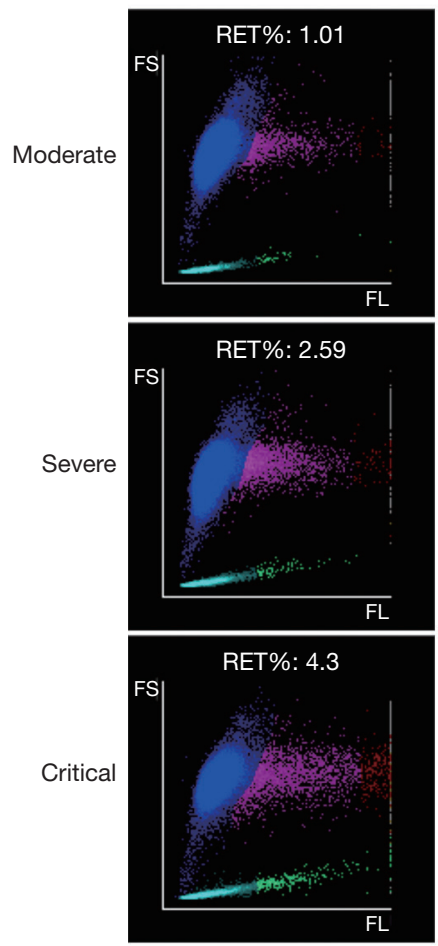

RBC scatter
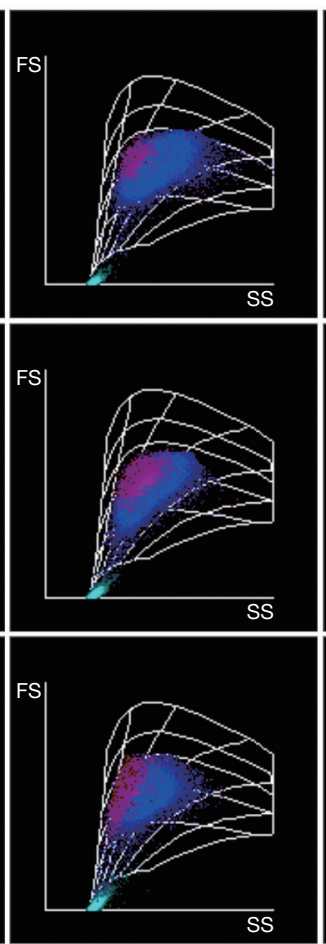

RBC V/HC scatter
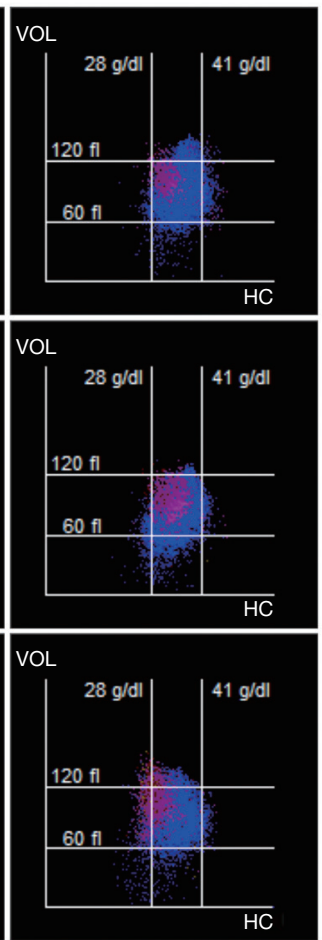

B RDW-SD

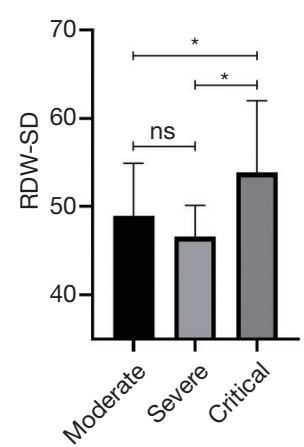

$\mathrm{D}$

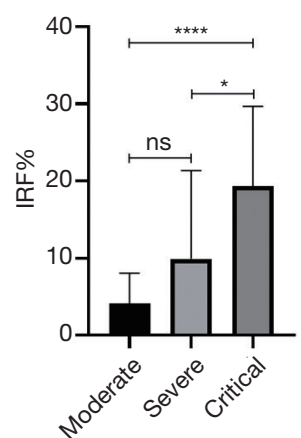

C RET\%

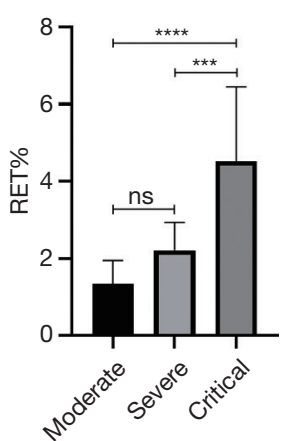

$\mathrm{E}$

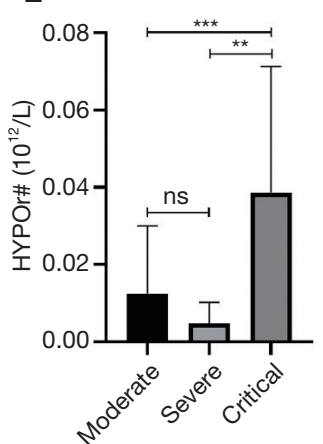

Figure 2 Differences of RET channel results in patients with different severity of COVID-19. (A) Scattergram of RET channel data from Mindray BC-6800 hematology analyzer. Blue scatters are RBCs, magenta, and the red scatter is the RETs, and cyan scatters are PLTs. (B,C,D,E) Comparison of parameters obtained from the RET channel for patients with different severities of COVID-19. Data are shown as the mean \pm SD. ${ }^{* * *}, \mathrm{P}<0.0001 ;{ }^{* * *}, \mathrm{P}<0.001 ;{ }^{* *}, \mathrm{P}<0.01 ;{ }^{*}, \mathrm{P}<0.05$. FS, forward scatter; SS, side scatter; FL, fluorescence; HC, hemoglobin concentration; VOL, volume; ns, nonsignificant.

hyperplasia in critical COVID-19 patients (Figure 2A). Comparisons of output parameters showed RDW-SD, RET\%, immature reticulocyte fraction (IRF\%), and hypochromic reticulocyte count (HYPOr\#) in critical patients were significantly higher than those in moderate and severe patients $(\mathrm{P}<0.05)$; however, those parameters were not significantly different between moderate patients and severe patients (Figure 2B,C,D,E). 


\section{Discussion}

Since the COVID-19 outbreak began in December 2019, there have been many reports on hematological parameters in patients with COVID-19. However, most reports have focused on the differences in hematological parameters in patients with different severities of COVID-19. There are few studies on the role of hematological parameters in predicting the prognosis of patients. In this study, a comparison of hematological test results of patients with different outcomes showed RDW might be a predictor of prognosis in patients with severe COVID-19 and that the increase in RET may be one cause for the increase in RDW.

This study included 98 patients with COVID-19 at Jingzhou Central Hospital and Central Hospital of Wuhan from January 20, 2020, to March 30, 2020. The prognostic analysis was performed with 43 patients diagnosed as severe at admission from January 20, 2020, to March 10, 2020. The data collected included the epidemiological information, clinical information, and hematological test results (without RET channel data) of the patients. The results showed that the increase in RDW might be a prognostic factor in critical patients. To investigate the causes of elevated RDW, we conducted a mechanism analysis with 55 patients with COVID-19 from March 12, 2020, to March 30, 2020, focusing on RET channel data in the hematological analysis of these patients.

Epidemiological and clinical information showed that the median age of the included patients was 59 [28-80] years old; the median age of the patients with a good outcome was 56 [28-79] years old, and the median age of the patients with a poor outcome was 67 [35-80] years old. Patients with a poor outcome were older than those with a good outcome $(\mathrm{P}<0.05)$, suggesting that elderly patients are more prone to a poor outcome. However, there were no significant differences in exposure history, comorbidities, symptoms, or signs between the two groups.

Currently, many group study laboratory tests only show the results according to the disease initial severity of patients. However, disease severity constantly changes throughout hospitalization. Therefore, this study separately classified each blood sample according to the condition of the corresponding patient at the time of sample collection. Patients who did not develop critical illness within 3 days after sample collection were included in the good outcome group, and patients who developed critical illness within 3 days after sample collection were included in the poor outcome group; patients with critical illness before sample collection were not included in the statistics. From the comparison of hematological parameters in the two groups, Lym\#, RBC, HGB, HCT, MCV, and $\mathrm{MCH}$ were significantly lower in the poor outcome group than in the good outcome group $(\mathrm{P}<0.05)$, RDW-CV and RDW-SD were significantly higher in the poor outcome group than in the good outcome group $(\mathrm{P}<0.0001)$. ROC curves showed RDW-SD was the best single parameter for predicting the prognosis of ill patients, with an AUC of 0.870 (95\% CI: 0.796-0.943). LDA was used to combine any two random routine hematological parameters linearly. Among the joint parameters generated, Lym\# \& RDW-CV had the most significant predictive efficacy, with an AUC of 0.928 (95\% CI: $0.852-1.000)$. When the cut-off value was 0.64 , the sensitivity and specificity of Lym\# \& RDW-CV were $95.7 \%$ and $83.3 \%$, respectively. Recent studies $(11,12)$ have reported that RDW can be a prognostic factor for infectious diseases (including sepsis and severe viral infection), which is consistent with the present study.

RDW shows the heterogeneity in RBC volume in peripheral blood. The higher the RDW is, the more significant the heterogeneity in RBC volume. RET channel testing of the blood samples was added for the patients in the mechanism analysis to investigate the cause of increased heterogeneity in $\mathrm{RBC}$ volume in severe patients with a poor outcome. The RET channel of the Mindray BC6800 hematology analyzer can detect the number, size, and hemoglobin concentration of RBCs using high-sensitivity laser scattering technology and can quantify and classify RETs using fluorescence detection. The results showed that the number of RETs was significantly higher in critical patients than in moderate patients and severe patients. Because the volume of a RET is more significant than a mature RBC, the increase in RETs may cause an increase in RDW.

Interestingly, the RBC V/HC scattergram showed RETs with low $\mathrm{HC}$ was significantly increased in critical patients. This trend was shown in the histogram of parameters as significantly increased HYPOr\# in the critically ill group, which may have occurred because the increased erythropoietin synthesis and active erythroid hyperplasia because of long-term hypoxia and the impaired HGB synthesis because of malnutrition or iron deficiency in critical patients resulted in low-HC RETs in the newly generated RETs. However, whether such low-HC RETs could a diagnostic marker of critical COVID-19 still requires further investigation. There is no report on the RET level in patients with COVID-19. However, some researchers $(13,14)$ have found immature RBCs in 
peripheral blood smears from patients with COVID-19, and an increase in RETs often accompanies immature RBCs in peripheral blood. Their findings indirectly support the findings in this study.

Because the number of RET significantly increased in critical patients, and the volume of RETs was more significant than mature RBCs, their MCV should also be increased. However, the MVC was lower in patients with a poor outcome than in patients with a good outcome in this study (Table 2). This finding suggests that the increase in numbers of RETs may not be the only cause of the increase in RDW in patients with COVID-19 with a poor outcome. It has been reported that before aggravation of infection symptoms, the level of oxidative stress in the body increases significantly, and the release of oxygen free radicals increases (15). Also, insufficient circulating nutrients in patients with a poor outcome may lead to an increase in RBC membrane instability, increasing RDW. All these factors might cause an increase in RDW and RETs and a decrease in MCV in patients with a poor outcome $(16,17)$.

Because the mechanism analysis included relatively numerous blood samples from ill patients (6 cases), it was difficult to group them according to prognosis. Therefore, for the mechanism analysis, statistical analysis was performed after grouping the blood samples according to disease severity. The conclusion from the analysis was that the significant increase in RETs in critical patients was only a probable reason for the increase in RDW in patients with a poor outcome. More patient samples will be collected to obtain more RET channel data for verification.

In summary, this study statistically analyzed the hematological test results of patients with COVID-19 in two hospitals and found RDW can be used as a predictor for the prognosis of severe patients and that an increase in numbers of RETs may be one cause of the increase in RDW.

\section{Acknowledgments}

We thank all patients who took part in this study. Thanks to all health-care workers in our hospital for their efforts in caring for these patients. Thanks to all people who work so hard to fight against the novel coronavirus pneumonia (COVID-19).

Funding: None.

\section{Footnote}

Reporting Checklist: The authors have completed the MDAR reporting checklist. Available at http://dx.doi.org/10.21037/ atm-20-6090

Data Sharing Statement: Available at http://dx.doi. org/10.21037/atm-20-6090

Conflicts of Interest: All authors have completed the ICMJE uniform disclosure form (available at http://dx.doi. org/10.21037/atm-20-6090). The authors have no conflicts of interest to declare.

Ethical Statement: The authors are accountable for all aspects of the work in ensuring that questions related to the accuracy or integrity of any part of the work are appropriately investigated and resolved. All procedures performed in this study involving human participants were in accordance with the Declaration of Helsinki (as revised in 2013). The Ethics Committee approved this study of Jingzhou Central Hospital and the Ethics Committee of the Central Hospital of Wuhan. As this study was a retrospective study, there was no patient's private data, including patient name, ID number, telephone, and address, were involved. Only demographic information and laboratory testing data of patients were collected and analyzed, so there is no informed consent in this study.

Open Access Statement: This is an Open Access article distributed in accordance with the Creative Commons Attribution-NonCommercial-NoDerivs 4.0 International License (CC BY-NC-ND 4.0), which permits the noncommercial replication and distribution of the article with the strict proviso that no changes or edits are made and the original work is properly cited (including links to both the formal publication through the relevant DOI and the license). See: https://creativecommons.org/licenses/by-nc-nd/4.0/.

\section{References}

1. China National Health Commission. Diagnosis and treatment of pneumonia caused by new coronavirus infection (version 7) (Mar 3, 2020). Beijing: China National Health Commission, 2020.

2. COVID-19 Dashboard by the Center for Systems Science and Engineering (CSSE) at Johns Hopkins University (JHU). Available online https://coronavirus.jhu.edu/map. html. 4/27/2020, 10:42:00 AM

3. Yang X, Yu Y, Xu J, et al. Clinical course and outcomes of critically ill patients with SARS-CoV-2 pneumonia 
in Wuhan, China: a single-centered, retrospective, observational study. Lancet Respir Med 2020;8:475-81.

4. Chen N, Zhou M, Dong X, et al. Epidemiological and clinical characteristics of 99 cases of 2019 novel coronavirus pneumonia in Wuhan, China: a descriptive study. Lancet 2020;395:507-13.

5. Qin C, Zhou L, Hu Z, et al. Dysregulation of Immune Response in Patients With Coronavirus 2019 (COVID-19) in Wuhan, China. Clin Infect Dis 2020;71:762-8.

6. Ruan Q, Yang K, Wang W, et al. Clinical predictors of mortality due to COVID-19 based on an analysis of data of 150 patients from Wuhan, China. Intensive Care Med 2020;46:846-8.

7. Bizzarro MJ, Conrad SA, Kaufman DA, et al. Infections acquired during extracorporeal membrane oxygenation in neonates, children, and adults. Pediatr Crit Care Med 2011;12:277-81.

8. Gong J, Ou J, Qiu X, et al. A Tool to Early Predict Severe 2019-Novel Coronavirus Pneumonia (COVID-19): A Multicenter Study using the Risk Nomogram in Wuhan and Guangdong, China. MedRxiv 2020. doi: https://doi.or g/10.1101/2020.03.17.20037515.

9. Sharma D, Dayama A, Banerjee S, et al. To Study the Role of Absolute Lymphocyte Count and RDW in COVID 19 Patients and their Association with Appearance of Symptoms and Severity. J Assoc Physicians India 2020;68:39-42.

Cite this article as: Wang C, Zhang $\mathrm{H}$, Cao X, Deng R, Ye Y, Fu Z, Gou L, Shao F, Li J, Fu W, Zhang X, Ding X, Xiao J, Wu C, Li T, Qi H, Li C, Lu Z. Red cell distribution width (RDW): a prognostic indicator of severe COVID-19. Ann Transl Med 2020;8(19):1230. doi: 10.21037/atm-20-6090
10. Qu R, Ling Y, Zhang YH, et al. Platelet-to-lymphocyte ratio is associated with prognosis in patients with coronavirus disease-19. J Med Virol 2020. doi: 10.1002/ jmv. 25767.

11. Hu ZD, Lippi G, Montagnana M. Diagnostic and prognostic value of red blood cell distribution width in sepsis: A narrative review. Clin Biochem 2020;77:1-6.

12. Wu J, Zhang X, Liu H, et al. RDW, NLR and RLR in predicting liver failure and prognosis in patients with hepatitis E virus infection. Clin Biochem 2019;63:24-31.

13. Mitra A, Dwyre DM, Schivo M, et al. Leukoerythroblastic reaction in a patient with COVID-19 infection. Am J Hematol 2020;95:999-1000.

14. Zini G, Bellesi S, Ramundo F, et al. Morphological anomalies of circulating blood cells in COVID-19. Am J Hematol 2020;95:870-2.

15. Diao B, Wang C, Wang R, et al. Human Kidney is a Target for Novel Severe Acute Respiratory Syndrome Coronavirus 2 (SARS-CoV-2) Infection. medRxiv 2020. doi: https://doi.org/10.1101/2020.03.04.20031120.

16. Li R, Rivers C, Tan Q, et al. The demand for inpatient and ICU beds for COVID-19 in the US: lessons from Chinese cities. medRxiv 2020. doi: https://doi.org/10.1101/2020.03 09.20033241.

17. Zhang FX, Li ZL, Zhang ZD, et al. Prognostic value of red blood cell distribution width for severe acute pancreatitis. World J Gastroenterol 2019;25:4739-48. 